\title{
Abstracts of Lectures at the Workshop
}

\section{Double-Stranded RNA-Induced Eosinophilic Airway Inflammation \\ Yoshiki Shiraishi ${ }^{\mathrm{a}, \mathrm{c}}$, Koichiro Asano a, c, Kyoko Niimi a, c,

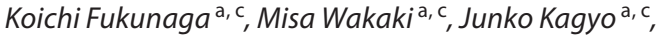 Takahisa Takihara a, c, Takeshi Nakajima a, Tsuyoshi Oguma a, Yusuke Suzuki ${ }^{a}$, Tetsuya Shiomi ${ }^{a}$, Koichi Sayama ${ }^{a}$, Eiji Ikeda ${ }^{\text {b, }}$ Hiroyuki Hirai ${ }^{\text {d, Masataka Nakamura }}{ }^{\mathrm{e}}$, Akitoshi Ishizaka ${ }^{\mathrm{a}, \mathrm{c}}$ \\ aDivision of Pulmonary Medicine, Department of Medicine, ${ }^{b}$ Department of Pathology, and 'Pfizer-Keio Research Laboratories, Shinanomachi Research Park, Keio University School of Medicine, Tokyo, dDepartment of Advanced Medicine and Development, Bio Medical Laboratories, Inc., Saitama, eHuman Gene Sciences Center, Tokyo Medical and Dental University, Tokyo, Japan}

Rationale: Respiratory RNA virus infection exacerbates respiratory symptoms, pulmonary function, and airway inflammation in asthmatic patients. We examined the effects of double-stranded RNA (dsRNA) synthesized during RNA virus replication on eosinophilic airway inflammation in vivo. Methods: Ovalbumin (OVA)-sensitized BN rats were exposed to aerosolized OVA, followed by intratracheal instillation of synthetic dsRNA, poly(I:C). Bronchoalveolar lavage (BAL) was performed $24 \mathrm{~h}$ after OVA exposure, and the profiles of cellular and humoral factors in BAL fluid were determined. Results: Poly(I:C) instilled at $16 \mathrm{~h}$ after OVA exposure significantly increased the number of eosinophils in BAL fluid and in lung tissue. In OVA-exposed lungs which exhibited an enhanced cyclo-ocxygenase-2 (COX-2) mRNA levels in alveolar macrophages, poly(I:C) induced a significant increase in $\mathrm{PGD}_{2}$ release in a COX-2-dependent manner. $\mathrm{PGD}_{2}$ instilled in the trachea alone enhanced BAL eosinophilia in OVA-exposed animals, and poly(I:C)-induced BAL eosinophilia was almost completely suppressed by the pretreatment with a dual antagonist against $\mathrm{PGD}_{2}$ receptor, $\mathrm{CRTH} 2$, and thromboxane receptor, TP, but not with a TP-specific antagonist. Conclusions: During allergic airway inflammation, dsRNA induced COX-2-dependent production of $\mathrm{PGD}_{2}$, which further recruited eosinophils into the airway via CRTH2 receptor. [This manuscript is preparing submission.]

\section{Involvement of $\mu$-Opioid Receptors in the Exacerbation of Airway Inflammation in a Murine Model of Allergic Asthma}

Kaori Okuyama a, Yoshie Arai a , Satoko Kajikawa ${ }^{a}$, Maya Naruse a, Gen Tamurac, Shinobu Sakurada b, Ichiro Sora ${ }^{a}$, Motoaki Takayanagi ${ }^{a}$, Isao Ohno a

Departments of apathophysiology and bPhysiology and Anatomy, Tohoku Pharmaceutical University, 'Department of Respiratory and Infectious Diseases and dDivision of Psychobiology, Department of Neuroscience, Tohoku University Graduate School of Medicine, Tohoku, Japan

Psychological stress has long been recognized to be associated with asthma exacerbation. However, the mechanisms linking stress to asthma are not well defined. Psychological stress upregulates the expression of endogenous opioids. The opioids stimulate the hypothalamus-pituitary-adrenal axis and sympathetic and adrenomedullary system, through the activation of $\mu$-opioid receptor (MOR) to release stress hormones, such as cortisol and catecholamines, respectively. These hormones can shift the immune response from Th1 to Th2 by the downregulation of Th1 cytokine expression. Therefore, we investigated whether MOR contributed to asthma exacerbation induced by psychological stress using a murine model of allergic asthma. Female C57BL/6 mice sensitized with ovalbumin (OVA) were exposed to restraint stress (RS) as psychological stress for 7 days consecutively. One day after the last RS, mice were given OVA inhalation. The contents of IL-4 and IL-5 and the numbers of total cells, eosinophils and lymphocytes in bronchoalveolar lavage fluids obtained from mice with RS were significantly higher than those from mice without RS. The effect of RS was abolished by the simultaneous administration of an MOR antagonist, and was not observed in MOR-deficient C57BL/6 mice. These findings suggest that MOR is involved in the psychological stress-induced exacerbation of allergic airway inflammation.

\section{KARGER}

Fax +41 613061234

E-Mail karger@karger.ch

www.karger.com
(C) 2007 S. Karger AG, Basel

$1018-2438 / 07 / 1435-0110 \$ 23.50 / 0$

Accessible online at: www.karger.com/iaa 